\title{
Control of a Semi-Circular Planform Wing in a "Gusting" Unsteady Free stream Flow II: Modeling and Feedback Design
}

\author{
Gilead Tadmor ${ }^{1}$ \\ Northeastern University, Boston, MA, 02115 \\ David R. Williams ${ }^{2}$ and Jesse Collins ${ }^{3}$ \\ Illinois Institute of Technology, Chicago, IL, 60616 \\ Tim Colonius ${ }^{4}$ \\ California Institute of Technology, Pasadena, CA, 91125 \\ and \\ Clarence W. Rowley ${ }^{5}$ \\ Princeton University, Princeton, NJ, 08544
}

\begin{abstract}
Active flow control has been demonstrated in Part I of this article to modify the lift, drag and pitching moments on a semi-circular wing during "gusting" flow conditions. The low aspect ratio wing, $A R=\mathbf{2 . 5 4}$, is mounted on a captive trajectory system that responds to the instantaneous lift force and pitching moment and the "gusting" flow is simulated by a 0.2 $\mathrm{Hz}$ oscillation of the free stream speed of the wind tunnel. The mean chord Reynolds number of the wing is 70,600. Active flow control occurs along the leading edge of the airfoil, which contains 16 spatially localized micro-valve actuators. Details of the experimental setup, a quasi steady state lift model and results involving open-loop proof of concept validation are provided in Part $I$ of this paper. Here we outline principles and considerations associated with close loop design that will be discussed in our talk.
\end{abstract}

\section{Introduction}

The research outlined in our two parts abstract is carried as part of a MURI research program whose purpose is to use feedback flow control to extend the range of aspect ratios, Reynolds numbers and angles of attack (AOA) for which high lift and high maneuverability can be achieved. Our work is motivated by the remarkable aerodynamic performance of bio-fliers wings, enabled by their stable leading edge vortex (LEV) that prevents stall at high angles of attack. To extend such capabilities from flapping to fixed wing flight we use pulsed-blowing actuators placed along the leading edge of a wing to modify the overall lift and pitching moment by controlling the development and interactions of the leading-edge and the tip vortex (LEV/TV) system. Part I of this abstract is used to introduce duty ratio modulation as an effective means to manipulate the produced lift and provide a proof of concept validation of the capability of this form of active flow control to counterbalance the effects of an unsteady free stream flow simulating wind gusts. Part I utilized a quasi-steady state lift model, which is ample for open-loop design utilizing harmonic balancing. Yet an essential ingredient of viable model-based feedback control is the correct representation of dynamic time constants ad delays in the response of the system. Elucidating these aspects is the goal of this part.

\footnotetext{
${ }^{1}$ Professor, Electrical \& Computer Engineering and Mathematics

${ }^{2}$ Professor, Mechanical \& Aerospace Engineering

${ }^{3}$ Graduate Student, Mechanical \& Aerospace Engineering

${ }^{4}$ Professor, Engineering and Applied Science

${ }^{5}$ Assoc. Professor, Mechanical and Aerospace Engineering
}

American Institute of Aeronautics and Astronautics 092407 

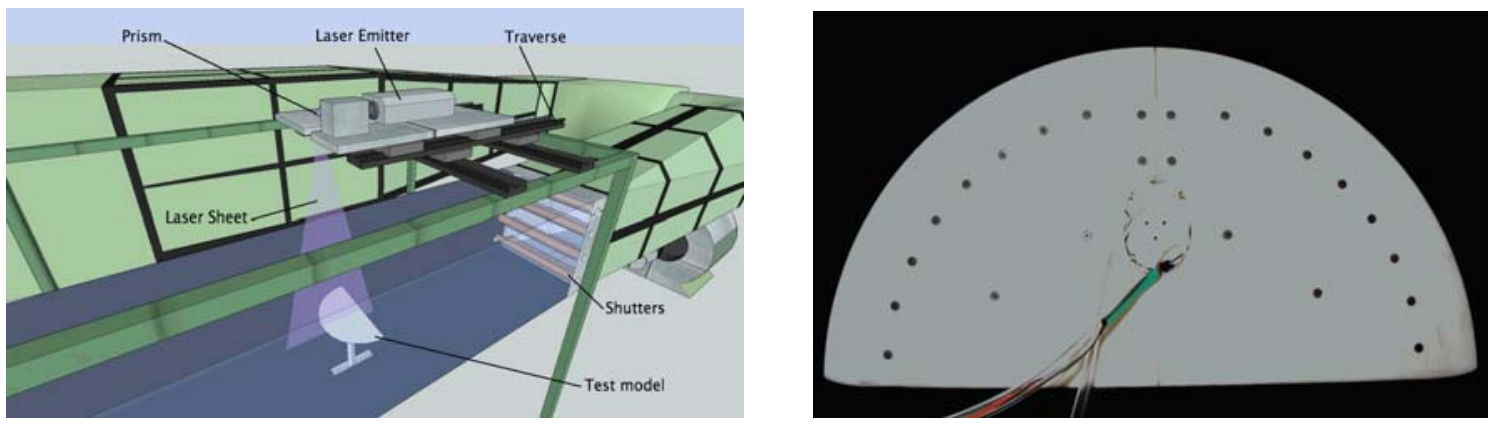

Fig. 1 - Left: The IIT - Fejer unsteady flow wind tunnel showing the semicircular airfoil model and a PIV system arrangement. Right: Bottom view of the 3D airfoil model, showing the force balance mounting holes (center 3 holes), air supply lines, micro-valve and pressure transducer connections.

\section{Experimental Setup and Actuation Impact}
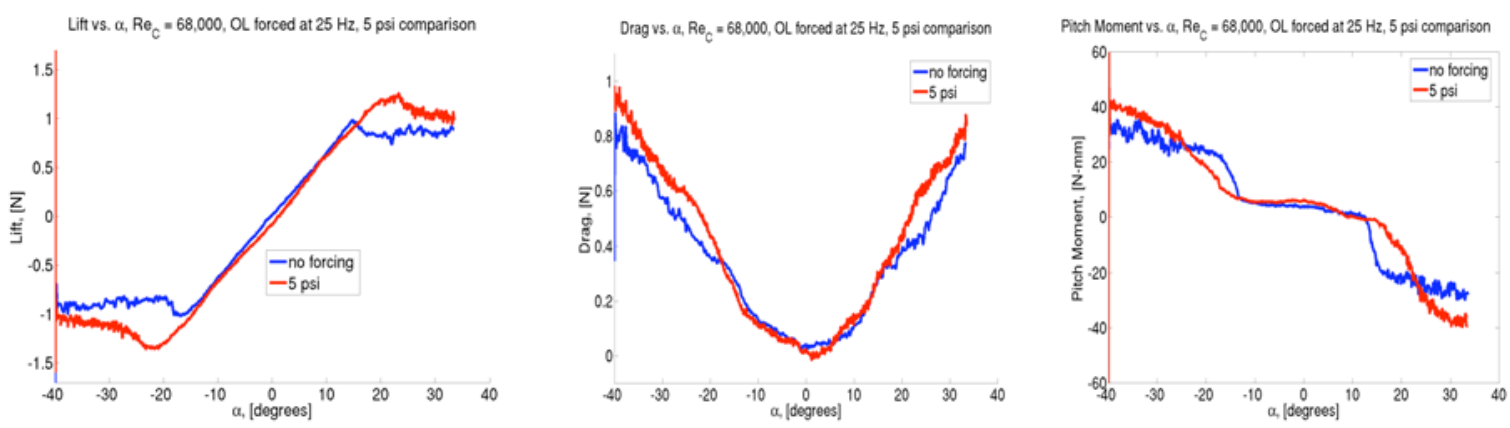

Figure 2. Lift (left), drag (center) and pitching moment (right) dependence on angle of attack, indicating the influence of $25 \mathrm{~Hz}$ open loop actuation at $\mathrm{Re}_{\mathrm{c}}=68,000$.

For completeness and ease of reading we provide a brief overview the experimental system that was discussed in more detail, in Part I. The semicircle airfoil model is tested in the Andrew Fejer Unsteady Flow Wind Tunnel, both shown in Fig. 1. The airfoil's centerline chord c (and radius R) is $203 \mathrm{~mm}$, hence a span of b $2 \mathrm{c}$ and aspect ratio of $\mathrm{b}^{2} / \mathrm{S}=8 / \pi=2.54$. Testings reviewed here were carried at a chord Reynolds number of $\operatorname{Re}_{\mathrm{c}}=68,000$. Free stream speed modulation at frequencies of up to $3 \mathrm{~Hz}$ is enabled by a computer controlled shutter at the downstream end of the test section. The model is mounted on a two-component vertical sting, actuated by Xenus servotubes. The model's height, pitch angle and pitch rate are regulated, using the sting actuation and a feedback control implemented on a dSPACE 1102 system. This configuration enables to simulate complex flight maneuvers in captive trajectories. The means for active flow control are provided by sixteen micro-valves for pulsed-blowing actuation, installed internally along the leading edge of the wing. At this stage all actuators are driven in phase, although testing of circumferentially varying actuation is also planned. Real time, on-board sensing is provided by two surface pressure taps at $\mathrm{x} / \mathrm{c}=0.4$ and $\mathrm{x} / \mathrm{c}=0.72$. The transient response of the leading edge vortex and the tip vortex system is documented by recording the two surface pressure signals, and the lift, drag and pitching moment using an ATI force 6-component balance system.

Pulsed, square wave actuation is applied in the experiments described here with a momentum coefficient of $\mathrm{C}_{\mu}=$ 0.0074 and a base period of $\mathrm{T}_{\mathrm{B}}=0.04 \mathrm{sec}$, of which the valves open for the first $0.5 \mathrm{~T}_{\mathrm{B}}=0.02 \mathrm{sec}$. This is equivalent to a $25 \mathrm{~Hz}$ in continuous pulsed operation. The lift, drag and pitching moment forces acting on the semi-circular wing are shown in Fig. 2 with and without leading edge actuation. The effect of the actuation is to delay stall beyond the AOA of $\alpha=15^{\circ}$, and to increase both the maximal lift coefficient (by some $20 \%$, just prior to stall) and the drag. To explore the benefits of actuation, this discussion is focused solely on the AOA of $19^{\circ}$, well in the post stall regime.

American Institute of Aeronautics and Astronautics 092407 
For the purpose of feedback control we need to produce a continuous range of lift increments. Pressure amplitude modulation is ineffectual, both when the range of lift increments and required hardware are considered, and modulation of the pulsing duty ratio is used instead. In the current implementation the duty ratio is defined as the number of time intervals of length $T_{B}$ during which pulse blowing takes place, out of a larger interval of length $T_{C}=15 T_{B}$. The reason for this selection will be clarified in the next section. Control policies are thus defined in terms of the time varying duty ratio command, denoted DTC, which is used as the control input. Figure 3 depicts the mapping from constant DTC inputs to the lift increment, with both experimental measurements at the 16 feasible quantization levels (the red curve), and a continuous approximation of that, clearly nonlinear mapping (the blue curve). The DTC that produces the median increment is used as a baseline enabling both positive and negative corrections in response to disturbances, such as gusts.

\section{Aerodynamic Transient Aspects of Actuation Response}

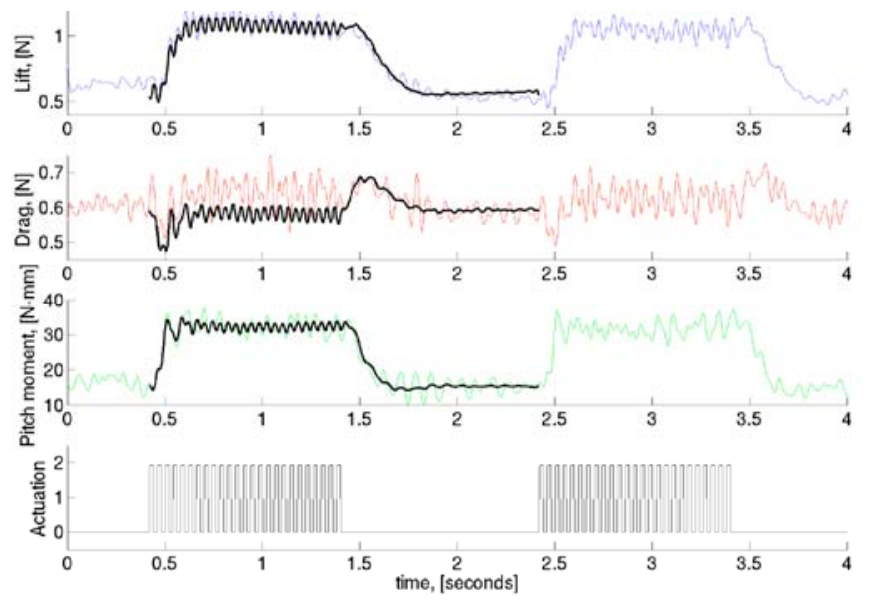

Figure 4. Transient response to $25 \mathrm{~Hz}$ actuation, switching $25 \mathrm{~Hz}$ pulsing actuation on and off at $1 \mathrm{sec}$ intervals. In each plot we see a generic time trace (in color) and a period averaged plot (solid, black). From top to bottom, plots include the produced lift, drag, pitching moment and the valve command. data measurements, both under actuation and in the un-actuated flow. These fluctuations are associated with the flow and release of vortical structures over the wing, and their frequency range is thus compatible with that of related flow dynamics, which is comparable with that of the pulsed actuation. The second aspect concerns the convergence rate once actuation is initiated and terminated, as well as the nonlinearity of the response, which has already been noted in the previous section. While the transient of large-scale characteristics is somewhat shorter at the initiation of the actuation than at its termination, both can be described with a time constant of roughly $0.125 \mathrm{sec}$ and settling of nearly $0.25-0.3 \mathrm{sec}$. These delays are particularly conspicuous in the otherwise fairly flat response of the drag force, where we see a lower frequency deep, at the inception of the actuation, and a substantial raise, after its cessation. An explanation of the latter is in the fact that while pulsed actuation gives rise to a strong LEV, 

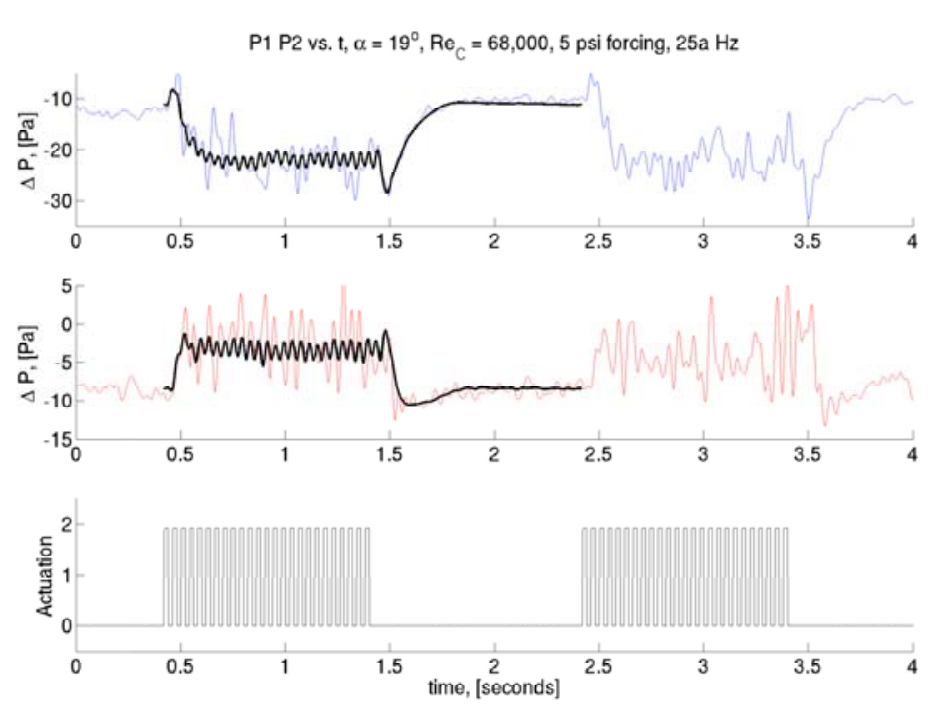

Figure 5. Transient response to $25 \mathrm{~Hz}$ actuation, switching $25 \mathrm{~Hz}$ pulsing actuation on and off at 1 sec intervals, complementing Fig. 5. In each plot we see a generic time trace (in color) and a period averaged plot (solid, black) of the front (blue) and bottom (red) surface mounted pressure taps.

characterization indicates a time delay. The slope of the actuation respons sec, essentially the same as in Figs. 4 and 5.

\section{Implications of Achievable Performance and Duty Ratio Actuation}

The picture unveiled in by Figs. 4-6 includes the of time delays and phase lags, as well as a coherent explanation $^{1}$ of their existence in terms of intrinsic properties of the aerodynamic response to pulsed actuation. That is to say, it is independent of control policies and will remain valid regardless of the selected feedback design. Consequently, the time constant of achievable in closed loop is practically bounded by intrinsic performance limitations, at nearly an order of magnitude longer than the aerodynamic delay. This translates to a bandwidth 0.5 $\mathrm{Hz}$ or lower.

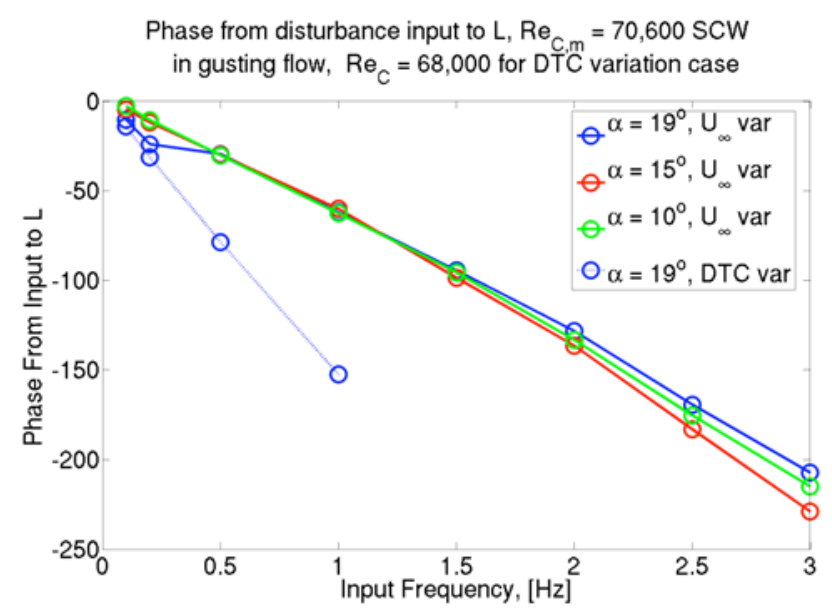

Figure 6. Input - Output phase shift lift dependence on sinusoidal input frequency: The 3 , nearly overlapping curves, represent the response to sinusoidal perturbations in $U_{\infty}$ at three values of the $\mathrm{AOA}$, and the stronger-tilted, single line represent the actuation response.

contributing to lift, it also regulates the LEV size by periodic shedding. Continued and weakening of the LEV precede The transient LEV growth contributes to the levated drag force.

Complementing the force moments depicted in Fig. 5, the transient readings by shown in Fig. 5. The pressure response to actuation is characterized by a pressure drop towards the front of the airfoil, consistent with a stronger LEV, followed with a short lag by a pressure rise more to the rear, consistent with a smaller / shorter LEV.

The observation made above are further supported by the describing function phase lag analysis, presented in Part I and repeated here for ease of reference, and presented here again as Fig. 6. It depicts the phase lag of the response to sinusoidal variations in the cree stream, as well as in the commanded DTC. The straight line of the phase lag in the frequency response

(1)


the averaging window defines an inherent lower bound on achievable closed loop time constant. From this perspective, a reasonable length of the averaging interval should be on par with the natural response time of the system. The selected value of $T_{C}=15 T_{B}=0.6 \mathrm{sec}$ is within that range.

\section{Models Appropriate for Feedback Design}

At the time scales of interest for small flyers, comparable in size and Re values to our experimental benchmark, dynamic models useful for feedback design must resolve both the response of the wing to aerodynamic forces and the aerodynamic response of the flow. In particular, the model must be able to predict the dynamic changes in the produced lift, drag and pitching forces that are associated with step changes in the incoming flow, such as those associated with more realistic gusts, fast pitching maneuvers and the like. Existing dynamic lift models ${ }^{2,3}$ can be calibrated to specific trajectories but are barely effective at a wide range of envisioned transients at the desire levels of temporal resolution. In our talk we shall present an alternative approach that builds on the mean field modeling approach developed by Noack et. $\mathrm{al}^{4-6}$ (see also ${ }^{7}$ ). By that approach the model resolves the tight coupling between the leading shedding harmonic and changes in the mean field. $\mathrm{In}^{5,6}$ we further demonstrated both the need for and the ease of accounting for mode deformation as the operating condition changes. Subsequently, in $^{8}$ (see also ${ }^{9}$ ) we tied lift dynamics to that of the leading POD and mean field modes. A design model based on this approach would tie mode deformation to mean field and operating conditions (e.g., AOA), and in turn, tie the aerodynamic forces to the individual modes.

\section{Acknowledgments}

We gratefully acknowledge the support for this work by the U.S. Air Force Office of Scientific Research (FA9550-05-0369 and FA9550-06-1-0373) with program manager Dr. Fariba Fahroo.

\section{References}

${ }^{1}$ Williams, D., and Collins, J., "Active Concentration of Vorticity Along the Leading Edge of a Semi-Circular Wing," American Physical Society-Div. Fluid Dynamics Abstract, Salt Lake City, Nov. Aug 2007.

${ }^{2}$ M. Goman and A. Khrabrov, "State space representation of aerodynamic characteristics of an aircrat at high angles of attack", J. Aircraft 31 (1994), 1109 - 1115

${ }^{3}$ Magill, J.C., Buchmann, M., Rixon, G., and McManus, K., "Dynamic Stall control Using a Model-Based Observer," J. Aircraft, Vol. 40, No. 2, 2003, pp. 355-362

${ }^{4}$ Noack, B., Afanasiev, K., Morzynski, M., Tadmor, G., and Thiele, F., "A hierarchy of low-dimensional models for the transient and post-transient cylinder wake," J. Fluid Mech., Vol. 497, 2003, pp. 335-363.

${ }^{5}$ M. Morzynski and W. Stankiewicz and B. R. Noack and R. King and F. Thiele and G. Tadmor, "Continuous mode interpolation for control-oriented models of fluid flow", in Active Flow Control, R. King Editor, Notes on Numerical Fluid Mechanics and Multidisciplinary Design V. 95, 260 - 278, Springer Verlag, 2007

${ }^{6}$ M. Luchtenburg and G. Tadmor and O. Lehmann and B. R. Noack and R. King and M. Morzynski, "Tuned POD Galerkin models for transient feedback regulation of the cylinder wake", AIAA Paper 2006-140 44th AIAA Aerospace Sciences Meeting and Exhibit, 2006

${ }^{7}$ S.G. Siegel, K. Cohen, J. Seidel, M. Luchtenburg and T. McLaughlin, "Low dimensional modelling of a transient cylinder wake using double proper orthogonal decomposition", J. Fluid Mech., 2008, in press.

${ }^{8}$ Luchtenburg, M., Gunther, B., King, R., and Tadmor, G., "A generalized mean-field model of the natural and highfrequency actuated flow around a high-lift configuration,” J. Fluid Mech., 2007, submitted.

${ }^{9}$ Protas, B and Wesfreid, J.E., "On the relation between the global modes and the spectra of drag and lift in periodic wake flows", Competes Rendus, 49 - 54 